\title{
Kernos
}

Revue internationale et pluridisciplinaire de religion grecque antique

$21 \mid 2008$

Varia

\section{Jean Rudhardt et la dikè}

\section{Évelyne Scheid-Tissinier}

\section{QpenEdition \\ Journals}

\section{Édition électronique}

URL : https://journals.openedition.org/kernos/1612

DOI : 10.4000/kernos. 1612

ISSN : 2034-7871

\section{Éditeur}

Centre international d'étude de la religion grecque antique

\section{Édition imprimée}

Date de publication : 1 janvier 2008

Pagination : 173-184

ISSN : 0776-3824

Référence électronique

Évelyne Scheid-Tissinier, « Jean Rudhardt et la dikè », Kernos [En ligne], 21 | 2008, mis en ligne le 01 octobre 2011, consulté le 24 août 2022. URL : http://journals.openedition.org/kernos/1612 ; DOI : https://doi.org/10.4000/kernos.1612 


\section{Jean Rudhardt et la dikè}

C'est d'abord sa filiation divine qui vaut à Dikè d'occuper une place privilégiée dans le dernier ouvrage de Jean Rudhardt, Thémis et les Horai. Recherche sur les divinités grecques de la justice et de la paix. Un ouvrage que son auteur désigne modestement dans ses remerciements comme "un petit livre », né du "souci d'améliorer notre compréhension de la piété hellénique », en partant d'un certain nombre de «divinités cosmiques et morales qu'il est nécessaire de prendre en considération pour comprendre comment les Grecs ont ressenti la présence du divin $»^{2}$. Les divinités ainsi convoquées pour être analysées étant Thémis et ses filles, les trois Hôrai qu'elle a eues de Zeus, telles qu'Hésiode les évoque dans sa Théogonie (901-902), c'est-à-dire Eunomia, Dikè et Eirénè, à savoir la Bonne Organisation, la Justice et la Paix.

Ces divinités ont la particularité de se présenter comme l'incarnation de notions abstraites, pourvues d'une portée philosophique et morale largement reconnue. Les idées d'harmonie, de justice et de paix s'attachent effectivement aux dénominations d'eunomia, de dikè et d'eirénè, lorsqu'elles se présentent sous la forme de noms communs. D'où le danger qu'il y aurait à vouloir aborder ces figures divines d'une manière qui serait chargée d'un faux bon sens. Selon une méthode qui les considérerait comme de simples allégories, ou qui verrait en elles le résultat de la divinisation secondaire de notions abstraites qui suscitent le respect des humains. Une approche qui se trouve d'emblée invalidée par l'ancienneté même de ces déesses qui ont leur place dans la généalogie hésiodique. Il faut donc définir un autre angle d'attaque qui permette de rendre compte du double aspect qui caractérise ces entités. Or, poursuit Jean Rudhardt, si l'on admet que des puissances «abstraites » comme la Justice ou la Paix «peuvent être figurées sous les traits d'une personne humaine, il ne serait pas exclu que les Grecs perçoivent aussi une présence divine dans une situation juste, dans l'état de paix, dans l'aspiration à la justice ou à la paix telles qu'ils les ressentent ordinairement $\aleph^{3}$. Une perspective qui, au-delà du problème posé par ces notions, permet également de mieux pénétrer un certain nombre de phénomènes mentaux. Ainsi « comment le raisonnement logique et la réflexion mythique

\footnotetext{
1 J. RuDHARDT, Thémis et les Horai, Recherches sur les divinités grecques de la justice et de la paix, Genève, Droz, 1999.

2 RudhardT, o.c. (n. 1), p. 11.

${ }^{3}$ RudHARDT, o.c. (n. 1), p. 13.
} 
se coordonnent-ils? Se développent-ils sur des plans différents ? Y a-t-il une logique mythique? » Enfin, dans la mesure où les Grecs ont perçu dans ces notions « des qualités divines », leur analyse fournit une occasion privilégiée de «mieux saisir la relation qui unit la morale à la religion dans la conscience hellénique $»^{4}$.

Un premier fait s'impose, qui est que si Themis est individualisée à la fois comme divinité et comme notion, c'est toujours en groupe que ses filles, les Hôrai, sont représentées et honorées ${ }^{5}$. Seul le nom spécifique dont elles bénéficient en tant que notions leur confère une personnalité et un rôle bien distincts qui se prêtent à une analyse plus précise. Laquelle analyse implique donc de laisser de côté l'aspect cultuel qui englobe les trois sœurs dans un même ensemble, pour s'attacher à ce que véhicule la «langue commune » qui considère chacune d'elles individuellement. Dans cet ensemble, c'est l'exploration de la dikèe, la notion qui possède la portée la plus large, que j’ai privilégiée en mettant en lumière tout à la fois la rigueur de l'approche qui a été suivie et l'originalité des résultats qu'elle a produits ${ }^{6}$.

\section{Les données de la tradition}

Dikè s'impose par le rôle que lui confèrent ses emplois les plus anciens, les emplois homériques et hésiodique qui l'associent fréquemment à themis, vouée comme elle à la désignation d'éléments qui se rapportent à l'ordre social. C'est ce qui avait, il y a maintenant plus d'un siècle, retenu l'attention de Gustave Glotz, qui pensait avoir identifié la nature du lien qui rattachait la dikè à la themis ${ }^{7}$. Une association qu'il faisait remonter à une époque antérieure à celle des cités et qui permettait de reconnaître clairement les rôles dont se trouvait respectivement investie chacune de ces notions. À cette époque, expliquait l'historien, le monde grec se composait d'une juxtaposition de clans familiaux,

${ }^{4}$ RUDHARDT, o.c. (n. 1), p. 14.

5 Rudhardt, o.c. (n. 1), p. 43-57 pour Themis et p. 82-96 pour les Hôrai, avec toutes les mentions de diverses statues et lieux de culte qui leur sont consacrés.

${ }^{6}$ L'étude de la Dikè occupe dans le livre de Jean Rudhardt, une partie du chapitre III luimême intitulé «Traits particuliers de chacune des Horai ». La deuxième partie de ce chapitre, sous le titre de "Dikèe», couvre les pages 104 à 145 et aborde deux points : «A. Le nom dikè̀» et «B. De la notion à la divinité »

7 G. Glotz, La solidarité de la famille dans le droit criminel en Grèce, Paris, 1904 [réimpr. New York, Arno Press, 1973]. Une vingtaine d'années plus tard, dans l'Introduction à sa synthèse sur La cité grecque. Le développement des institutions, Paris, 1928 [réimpr. Albin Michel, 1968] (L'Évolution de l'bumanitê), p. 14-15, Glotz réaffirme encore le rôle joué, dans les débuts du monde grec, par le genos qui « en ce temps-là, avait seul une organisation solide et durable. [...] Le groupe ainsi formé jouit d'une indépendance complète et n’admet aucune limite à sa souveraineté ». La réalité du genos a été depuis, on le sait, sévèrement contestée et complètement réévaluée par les études de F. BOURRIOT, Recherches sur la nature du genos. Étude d'bistoire sociale athénienne, période archä̈que et classique, Lille, 1976 et de D. Roussel, Tribu et cité, Paris / Besançon, 1976. 
les genè, qui regroupaient les hommes de même sang et coexistaient sans qu'il existe aucune autorité supérieure qui soit en mesure de les contrôler. Chacun de ces groupes, chaque genos se gérait donc lui-même et assurait sa paix intérieure au moyen de la themis, des règles de droit qui s'exerçaient à l'intérieur de la famille et se trouvaient légitimées par la religion. C'est en fonction de ces règles que l'individu qui portait atteinte à l'un des membres de son clan ou à ses biens, était considéré comme coupable et puni. La crainte de la punition garantissait ainsi l'unité et la solidarité indispensables à la survie du genos. Dans la gestion des rapports entre familles, entre genè, c'est en revanche la dikè qui prévalait, c'est-à-dire le principe des représailles et de la vengeance ${ }^{8}$. La dikè se présente par conséquent comme l'élément qui assure la gestion des mécanismes qui régulent les rapports des humains entre eux, par opposition à la themis, cette loi imposée d'en haut et qui commande le respect.

Le rôle dont s'est trouvée ainsi dotée dikè a marqué une étape importante. Reste la difficulté qu'il y a à concilier cette intuition avec les données du lexique et celles de l'étymologie. Les textes prêtent en effet à ce substantif plusieurs types d'emplois entre lesquels il n'est pas aisé de dégager une logique. Si l'on se réfère aux dictionnaires, en l'occurrence le Liddell-Scott-Jones, on trouve pour dikè un premier sens, celui de « custom, usage », d'où découle l'emploi adverbial classique de dikèn, "à la manière de ». Le deuxième sens est celui de "order, right », c'est-à-dire « ce qui relève de l'ordre, du juste ». Il y a enfin un troisième sens, celui de «jugement rendu, décision judiciaire». Si les contenus des rubriques deux et trois s'inscrivent effectivement dans le contexte juridique ou judiciaire qui est celui dans lequel on rencontre le plus souvent le terme dikè, le contenu de la première rubrique est moins aisé à cerner.

L'étymologie par ailleurs n'est pas d'un grand secours. É. Benveniste9 reste tributaire de la thèse de Gustave Glotz et dans son souci de soutenir la théorie développée par l'historien, il se réfère à la racine indo-européenne *deik-, qui a donné le latin dico, "dire » et le grec $\delta \varepsilon i x v u \mu l$, « montrer ». Cette racine pourrait signifier : " montrer par la parole », ce dont témoigne le composé latin judex où *deik- est conjoint à jus pour désigner « celui qui montre avec autorité », c'est-àdire le juge, seul habilité à dicere jus, à « dire le droit ».

Il y a une bonne trentaine d'années, Michael Gagarin dans les deux études particulièrement fouillées qu'il consacre à l'emploi de dikè à l'époque archaïque ${ }^{10}$, s'était heurté au même problème. Sceptique à l'égard de l'étymologie proposée par Benveniste, il choisit de se rallier à l'étymologie retenue en 1950 par Palmer qui fait lui aussi remonter le susbstantif dikè à la même racine indo-

8 GLOTZ, o.c. (n. 6), p. 67-68.

${ }^{9}$ É. Benveniste, Le vocabulaire des institutions indo-européennes, Paris, 1969, II, p. 111.

10 M. Gagarin, "Dikè in the Works and Days », CPh 68 (1973), p. 81-94; "Dikè in Archaic Thougth », CPh 69 (1974), p. 186-197. 
européenne * deik-. ${ }^{11}$ Mais il considère que ce substantif a possédé dès l'origine deux significations distinctes, qui se sont ensuite développées parallèlement. Dikè désignerait d'une part « le signe, la marque, la caractéristique », d'autre part « la limite, la ligne de division ». D'où une double filiation. La première qui conduit au sens de "usage, caractéristique», attestée dans la première série d'énoncés que retiennent les dictionnaires. La seconde qui conduit à l'idée "d'un arrangement» ou "d'une décision», obtenus en créant une ligne de séparation, une limite entre deux individus en conflit. Deux significations de dikè qui se sont développées chacune de leur côté et ont fini par n’avoir plus rien en commun. Si bien que Michael Gagarin, renonce à reconstituer une impossible unité de sens et choisit de privilégier la dimension de dikè qui lui paraît fondamentale, c'est-à-dire celle qui possède une portée tout à la fois juridique et judiciaire ${ }^{12}$.

Confronté à cet état des lieux, Jean Rudhardt ${ }^{13}$ commence par constater l'impossibilité qu'il y a à trouver, y compris dans les sources les plus anciennes, une base qui conforte la théorie évolutive jadis formulée par Gustave Glotz concernant l'origine de la dikè. Ce dont témoigne par exemple le fait que chez Hésiode dikè s'applique déjà aux décisions prises par les rois corrompus pour régler le différend qui oppose le poète à son frère Persès ${ }^{14}$. D'où il faudrait conclure qu'à cette époque dikè avait déjà perdu sa spécificité et pouvait s'appliquer aux relations intrafamiliales. Le rapprochement opéré par Benveniste permet néanmoins de rendre compte du " caractère normatif », fréquemment véhiculé par dikè dans les différents types d'emplois qui en sont faits. Il reste que l'exploitation systématique de l'ensemble de ses emplois, fournit seule une chance de retrouver l'unité perdue de ce substantif. C'est sur cette recension que Jean Rudhardt souhaite s'appuyer, fidèle à sa méthode qui est de considérer que rien ne peut nous être intelligible, que ce soit dans l'étude de la pensée ou dans celle de la religion « hors de la langue employée par les hommes qui les ont vécues $»^{15}$.

11 L.R PALMER, «The Indo-European Origins of Greek Justice », TPhS (1950), p. 149-168.

$12 \mathrm{~J}$. Rudhardt a la sagesse de ne pas s'attarder sur une étymologie qui, en l'occurrence, peine à offrir une base solide. Il est permis de penser que L. Gernet était arrivé à une conclusion similaire, lorsqu'il faisait remarquer dans son étude de 1955, «Sur la notion de jugement en droit grec », Droit et Société en Grèce ancienne, Paris, 1955, p.61-81, p. 62, n. 1, que "quand une étymologie est en cause, on est justifié à ne pas la prendre pour point de départ ».

13 RudhardT, o.c. (n. 1), p. 104-106.

14 Hésiode, Travaux, 34-39.

15 RudhardT, o.c. (n. 1), p. 160. C'est sur cette profession de foi méthodologique que se termine le livre. 


\section{La dikè individuelle}

L'examen des emplois qui relèvent de la première rubrique, celle pour laquelle le Liddell-Scott-Jones donne le sens de "custom, usage», fournit un premier élément, qui loin d'être marginal pour la compréhension de dikèe, offre au contraire un point de vue inattendu qui permet de penser la cohésion de l'ensemble. Dans un certain nombre de contextes dikè désigne en effet ce qui est le «lot convenant à un individu» ou à un groupe d'individus. Ou plus exactement « ce qui revient de droit à un individu, sa part, son droit ». Il s'ensuit que dikè peut désigner aussi bien ce qu'on attend d'un individu que ce qui lui est dû. C'est ce qui ressort ainsi des emplois bien connus dans lesquel dikè se trouve associé à un génitif pluriel qui désigne un ensemble d'individus. Il est ainsi question :

- «du lot des mortels », $\alpha u ̈ \tau \eta ~ \delta i ́ \varkappa \eta ~ \varepsilon ̇ \sigma \tau \iota ~ \beta \varrho o \tau \tilde{\omega \nu, ~ u n e ~ f o i s ~ q u ' i l s ~ s o n t ~ m o r t s ~}$ (Od. XI, 218),

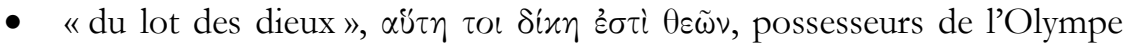
(Od. XIX, 43),

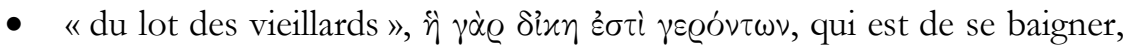
de manger et de dormir (Od. XXIV, 255).

En définissant ainsi le type de comportement qui caractérise telle ou telle catégorie d'individus, ce type d'énoncé peut servir à véhiculer une norme. Pénélope rappelle ainsi le comportement exemplaire d'Ulysse, qui, tout le temps qu'il a été à Ithaque, n'a jamais rien dit ni rien fait d'injuste contre personne, ce

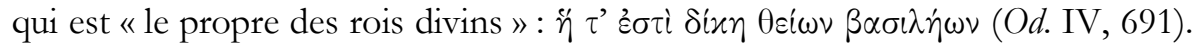
En revanche, la reine s'étonne, à un autre moment, du comportement des hommes qui l'entourent: "cette dikè n'est pas celle qu'avaient autrefois les

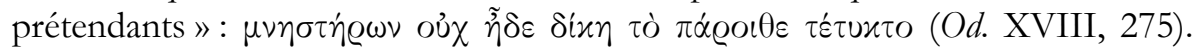
Une façon de désapprouver la dikè,, «la manière d'être » de ces gens, qui n'est pas conforme à celle que doivent avoir des hommes qui se trouvent engagés dans une compétition pour la main d'une femme. Ce qui, comme le relève justement Jean Rudhardt ${ }^{16}$, signale la transgression commise par les prétendants et laisse par là-même présager le châtiment qui en sera la conséquence.

Dikè définit de la même manière ce à quoi chacun a droit en fonction de sa position et qu'il peut donc revendiquer. Ce « lot», cette «part» qui leur revient, c'est précisément ce que les morts qui se pressent autour de Minos dans les Enfers cherchent à obtenir de lui. Ulysse évoque ainsi les gens qui demandent au roi «leurs dikai, les uns debout, les autres assis, dans la demeure d'Hadès aux larges portes $\gg^{17}$. On ignore de quoi dépend la nature de ces dikai. De la position qui était celle des défunts sur terre? De leur conduite pendant leur

\footnotetext{
${ }^{16}$ RUDHARDT, o.c. (n. 1), p. 112.

${ }^{17}$ Homère, $\mathrm{Od}$. XIV, 59-61.
} 
vie ? Tout en proclamant un droit, la dikè pose des limites. Dans l'Athènes de Solon par exemple, la nature de la dikè qui revient à chacun dépend de la position sociale qui lui est reconnue. Lorsqu'il évoque son œuvre, le législateur

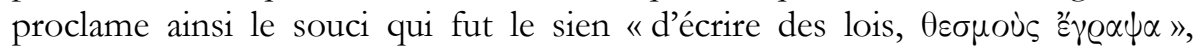
"pareillement pour le pauvre et pour le notable, $\delta \mu o i \omega \varsigma \tau \tilde{\varphi} x \alpha x \tilde{\varphi} x \dot{\alpha} \gamma \alpha \theta \tilde{\varphi}$ ", « en

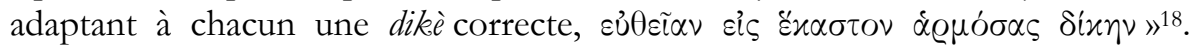
Donner à chacun ce qui lui revient ne signifie effectivement pas, dans l'Athènes archaïque, offrir à tout le monde la même part dans la gestion de la cité.

\section{Les moyens d'action de la dikè et leur raison d'être}

La protection de cette dikè est assurée par ce que Jean Rudhardt appelle «la dikè objective » et "la dikè subjective »19, qui sont intimement liées et qui relèvent l'une et l'autre de la deuxième rubrique, celle que le Liddell-Scott-Jones définit comme appartenant au domaine de ce qui est « order, right », c'est-à-dire de « ce qui relève de l'ordre, du juste ».

Cette dikè à double visage gère les conséquences positives ou négatives engendrées par les actions humaines. L'Électre d'Eschyle souhaite ainsi «que les meurtriers meurent à leur tour sous l'effet de la dikè̀ ». Ailleurs, les Choéphores évoquent la dikè qui «est venue enfin frapper les Priamides $»^{20}$. Il s'agit en l'occurrence de la chute de Troie, destinée à punir la faute commise par Pâris, lorsqu'il a enlevé Hélène. Dans les Sept contre Thèbes (444), Eschyle fait dire à Étéocle que celui qui méprise les avis de Zeus est destiné à être en retour frappé par le dieu. «La foudre chargée de feu s'abattra sur lui accompagnant la dikè. » Dans tous les cas, la dikè semble incarner "une puissance immanente à l'histoire ». Elle est évoquée de la même manière par Solon, lorsque le poète affirme que tout en désirant posséder des richesses, il refuse cependant de les

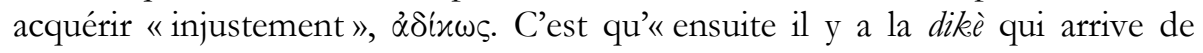
toute façon $»^{21}$. La dikè se présente donc comme une force autonome qui intervient pour punir le coupable. C'est en cela que consiste la dikè « objective». Un châtiment, une vengeance qui vient frapper en retour celui qui, si l'on se réfère au texte de Solon, a attaqué le premier autrui. Celui qui a agi «injustement», $\alpha \dot{\delta} i x \omega \varsigma$, en s'en prenant à la dikè de sa victime. Laquelle s'est trouvée d'une manière ou d'une autre lésée. Soit parce qu'elle a été privée de la vie dans le cas des personnes assassinées, soit parce qu'elle a été insultée dans le cas de la divinité dont la puissance est mise en doute, soit enfin parce qu'elle a été spoliée

\footnotetext{
${ }^{18}$ Solon, fr. 30, 18-20 (éd. Gentili-Prato).

${ }^{19}$ RUDHARDT, o.c. (n. 1), p. 112.

${ }^{20}$ Eschyle, Choéphores, 144 et 935.

${ }^{21}$ Solon, fr. 1, 7-8 (éd. Gentili-Prato).
} 
par celui qui était guidé par le désir des richesses ou par l'amour d'une femme qui appartenait à un autre.

Ce qui revient à dire que la dikè «objective» vient protéger la dikè dont bénéficie chaque individu, celle qui désigne «le lot» de chacun, ce à quoi il a droit. Toute agression suscite ainsi inévitablement une réaction en retour. Le mécanisme ainsi invoqué par Jean Rudhardt renvoie au schéma autrefois mis en évidence par Louis Gernet. Lequel avait été amené, en explorant la genèse de la notion de délit, à opérer une analyse précise de l'ensemble lexical constitué par dikè / adikein / adikia et adikèma. Puis à reconstituer la logique qui rend inéluctable l'irruption de la riposte destinée à compenser l'injustice ou le tort qui ont été dans un premier temps commis. Dans ce schéma, celui qui «porte atteinte à la dikè̀, $\alpha \delta(x \varepsilon \tilde{\imath}$, rompt un équilibre et introduit un désordre dont il porte la responsabilité. Ce que signale l'emploi très fréquent qui est fait de l'expression

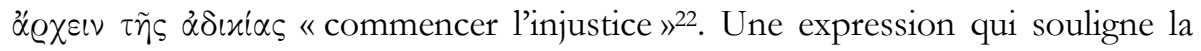
culpabilité que porte «celui qui a pris l'initiative du désordre, de la rupture d'équilibre».

L'historien du droit grec en avait déduit que le substantif dikè possèdait une dimension qui dépasse notre conception juridique ou morale de la justice. Une dimension dont on ne peut mesurer la portée qu'en la replaçant dans l'espace plus large et plus ancien «d'une représentation religieuse de l'équilibre», ellemême liée à la conception d'un ordre cosmique dont dépendent à la fois les choses de la nature et les affaires humaines ${ }^{23}$. Une vision, conclut de la même manière Jean Rudhardt que l'on trouve attestée dans la philosophie d'Anaximandre aussi bien que dans celle d'Héraclite. Lequel « appelle Dikè un principe régissant tout ce qui se produit dans l'Univers », un principe qui « agit parmi les hommes » et qui « agit aussi sur les entités cosmiques »24. Ce dont témoigne son précepte : «Le Soleil ne dépassera pas les mesures qui lui sont assignées, sinon, auxiliaires de Dikè, les Érinyes le découvriront $»^{25}$.

La dikè dite «subjective» est l'autre aspect de cette force active. Elle est "présente à l'esprit des hommes» et «influence leurs conduites». Elle est ancrée chez les humains comme un principe inné qui leur indique la voie du « juste ». Jean Rudhardt insiste sur le souci dont témoignent fréquemment les humains d'agir en ayant le droit de leur côté. L'Oreste d'Eschyle, rempli de doutes, demande ainsi anxieusement à Apollon s'il a bien tué sa mère «avec

22 L. GERnET, Recherches sur le développement de la pensée juridique et morale en Grèce, Paris, $2001^{2}$ [1917], p. $62-68$ et p. 64-65 où se trouvent mentionnées un certain nombre d'occurrences de

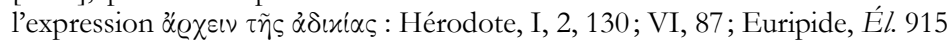

23 Gernet, o.c. (n. 18), p. 62. Voir sur ce même propos les développements de G. Vlastos, "Equality and Justice in early Greek Cosmology », CPh 42 (1947), p. 156-178, repris dans Studies in Greek. Philosophy, Princeton, 1995, I, p. 57-88.

${ }^{24}$ RudhardT, o.c. (n. 1), p. 142.

${ }^{25}$ Héraclite, 22 B 94 (éd. Diels-KRANZ ), cité par Rudhardt, o.c. (n. 1), p. 142. 


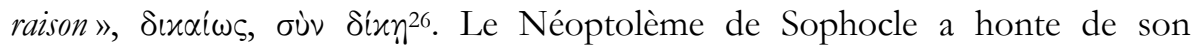
comportement à l'égard de Philoctète et veut lui restituer les armes que ce dernier lui a cédées. "Je les possède pour les avoir acquises de manière hon-

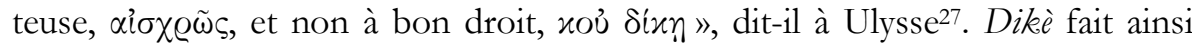
partie de ces notions qui habitent l'esprit des Grecs et sont antérieures aux spéculations philosophiques ${ }^{28}$. Cette «conscience de la dikè̀ » est propre à l'espèce humaine.

Elle est, affirme Hésiode avec force ${ }^{29}$, ce qui différencie les hommes des animaux, qui se dévorent entre eux parce qu'ils ne connaissent pas la dikè, cette manière de vivre, ce nomos que Zeus a donné aux hommes et qui permet à la société humaine de prospérer en vivant dans la paix. Sans dikèe, il ne peut exister de cohésion sociale. Respecter la dikè, si l'on suit jusqu'au bout la pensée de Rudhardt, cela revient pour l'homme à suivre cette parcelle de conscience divine dont Zeus lui a fait présent et qui lui permet de s'élever au-dessus des animaux. "À la fois respectable et mystérieuse, touchant à ce qui définit la condition humaine, et lui confère un sens, elle s'impose à la conscience des Grecs comme une force divine $»^{30}$. On conçoit alors que la Dikè soit représentée comme une fille de Zeus, comme une émanation de son père. Une fille qui, selon l'évocation qu'en fait Hésiode, court se réfugier auprès de Zeus, lorsqu'elle est offensée par les paroles ou les actes injustes des hommes et lui dénonce les coupables ${ }^{31}$. Ce qui provoque la colère du dieu, protecteur de Dikèe. Il fait alors payer le peuple ${ }^{32}$ et dévaste avec ses pluies les terres des hommes qui méprisent la justice ${ }^{33}$.

Cette dimension divine de la dikè se retrouve dans le mythe de Protagoras ${ }^{34}$, à travers lequel Platon évoque les origines de la civilisation en reconnaissant à la dikè jointe à l'aidôs un rôle décisif dans la mise en place des règles sociales. Il fut un temps en effet où les hommes vivaient dans la confusion et la violence. Ils ne cessaient «de se faire du tort les uns aux autres », adikein allèlous, ignorants de l'art politique, jusqu'au moment où Zeus leur a envoyé Hermès porteur de «l'aidôs (le respect de soi-même et celui des autres) ${ }^{35}$ et de la dikè, afin que leurs

\footnotetext{
${ }^{26}$ Eschyle, Euménides, 610, 612.

27 Sophocle, Philoctète, 1234.

${ }^{28}$ RUDHARDT, o.c. (n. 1), p. 155.

${ }^{29}$ Hés., Trav., 274-280.

${ }^{30}$ RUdHARDT, o.c. (n. 1), p. 133.

${ }^{31}$ Hés., Trav., 248-260.

32 Hés., Trav., 260.

${ }^{33}$ Hom., Il. XVI, 386-388.

${ }^{34}$ RudHARDT, o.c. (n. 1), p. 132-133.

35 Voir l'étude de L. CaIRns, Aidôs. The Psychology and Ethics of Honour and Shame in Ancient Greek Literature, Oxford, 1993, et celle de J. RUDHARDT, "Quelques remarques sur la notion d'aidôs», in Kĩr roı. De la religion à la philosophie. Mélanges offerts à André Motte, Liège, 2001 (Kernos, suppl. 11), p. 1-21.
} 
cités connaissent une belle ordonnance et des liens d'amitié propres à assurer leur cohésion $»^{36}$.

Cette dikè subjective "s'associe dans l'esprit des Grecs aux qualités que l'adjectif dikaios exprime ${ }^{37}$. Un homme mérite d'être qualifié de dikaios lorsqu'il se montre sensible aux exigences de la dikè et soucieux d'en respecter les obligations. Ce qui signifie notamment qu'il est attentif à ce qui revient à chacun en fonction de ses droits. Dans l'Odyssée, sont ainsi considérés comme dikaioi les hommes qui accueillent les étrangers en leur donnant l'hospitalité qu'ils sont en droit de recevoir ${ }^{38}$. Dans l'Iliade, à l'occasion de la cérémonie publique qui sanctionne la réconciliation entre Achille et Agamemnon, Ulysse qui a pris en charge l'organisation de la procédure, énumère toutes les démarches qu'Agamemnon doit accomplir pour satisfaire l'honneur blessé du héros. Après quoi le roi d'Ithaque conclut en s'adressant d'abord à Achille. Tout cela doit se faire, lui dit-il, " afin que rien ne te manque de ce qu'exige la dikè, ív $\alpha \mu n^{\prime}$

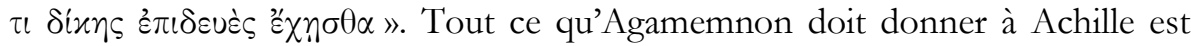
effectivement de l'ordre de la réparation. Dikè, désigne alors la compensation réparatrice que la victime est en droit de recevoir pour que soit restauré l'équilibre qui a été dans un premier temps compromis à ses dépens. Hésiode fait un emploi très semblable de dikè lorsqu'il évoque la réparation que l'agresseur propose de lui-même à sa victime. Dans ce cas, conseille Hésiode à Persès, si un ami qui t'a fait du tort " cherche ensuite à te ramener à son amitié et veut t'offrir

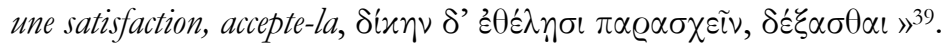

Dans la scène de réconciliation de l'Iliade, Ulysse poursuit son discours en s'adressant ensuite à Agamemnon : «Atride, pour toi, désormais sache être plus

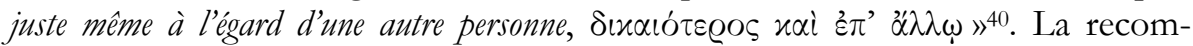
mandation ainsi faite au roi est destinée à l'inciter à savoir se montrer à l'avenir plus respectueux des droits d'autrui en évitant de léser quelqu'un d'autre comme il l'a fait pour Achille. Le respect de la dikè, l'attention portée aux droits d'autrui, exige effectivement une attitude qui met en œuvre la vigilance et le respect. On peut poursuivre dans cette direction en relevant qu'il s'agit d'une conception du juste, du dikaion, qui ne renvoie pas à une conception platonicienne d'une pure idée de la justice, mais plutôt à la conscience qu'un équilibre doit être maintenu entre les membres d'une même communauté qui possèdent le même statut. Cet équilibre qui garantit à chacun le respect de ce qui lui est dû

36 Platon, Protagoras, 322b-d.

37 RudhardT, o.c. (n. 1), p. 125 présente les choses d'une manière qui met l'accent sur la puissance agissante de dikè : "Le dikaios est celui que la dikè protège ou favorise. » Il rappelle par ailleurs que c'est sur dikeaios qu'est forgé le substantif dikaiosunè, qui désigne à l'époque classique « la qualité propre aux hommes justes ».

${ }^{38}$ Hom., Od. IX, 175.

${ }^{39}$ Hés., Trav., 712-713.

${ }^{40}$ Hom., Il. XIX, 179-181. 
est aussi le garant de la paix sociale. C'est la conception la plus traditionnelle du juste, celle que formule Polémarque, dans la République, lorsqu'il répond à Socrate qui lui demande une définition de la justice : «Ce qui est juste c'est de

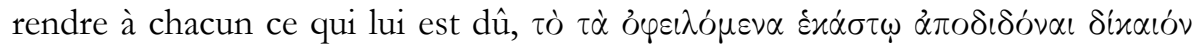

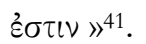

Si l'on admet que le rôle de la dikè subjective est de permettre à l'individu de distinguer et de respecter le «juste », le dikaion, ${ }^{42}$ c'est-à-dire de faire la part de ce qui lui revient et de ce qui revient aux autres, on saisit la portée de la recommandation qu'Hésiode adresse avec insistance à son frère Persès

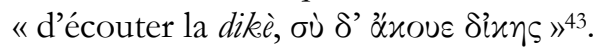

\section{La portée judiciaire de la dikè}

Dans les péripéties de la vie sociale cependant, il est fréquent que chacun se laisse guider par son intérêt personnel, qu'il n'ait pas toujours une perception équitable de ce à quoi il a droit, ou qu'il ne parvienne pas à faire reconnaitre ce droit par les autres. C'est le propos même des tragédies. C'est aussi la source de conflits dont la résolution est confiée aux arbitres ou aux juges. Ce qui confère à dikè la dimension judiciaire qu'on lui connait. La dikè et les dikeai désignent alors les décisions de justice qui sont rendues par les juges ou les arbitres auxquels les humains viennent soumettre leur différend.

«La dikè subjective, relève Jean Rudhardt, trouve son accomplissement dans une formule prononcée, dans un jugement, dans un verdict $»^{44}$. La dikè qui est ainsi émise résulte de l'opération qui est désignée par le verbe dikaz̨ein que nous traduisons par « juger» mais dont Ugo Paoli d'abord et Louis Gernet ensuite 45 ont montré qu'il recouvrait en fait une opération qui relevait moins de l'analyse intellectuelle que de la prise de décision. Le ou les juges sont invités à prendre

41 Platon, République I, 331d.

42 Rudhardt, o.c. (n. 1), p. 125 rappelle que to dikaion désigne chez Platon «l'idée de justice, de l'essence dont participent toutes les choses justes (Phédon, 65d, 75c) ». "C'est déjà sur la nature $\mathrm{du}$ dikaion que les pythagoriciens s'interrogent. »

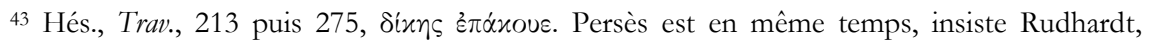
exhorté à s'abstenir de l'hubris. Le rôle joué par la dikè dans la régulation des rapports humains et dans le maintien du contrat social, par opposition à la force destructrice de l'bubris, se trouve mis en évidence de la même manière dans l'étude que J.-P. VERNANT a consacrée au mythe des races : "Le mythe hésiodique des races. Essai d'analyse structurale », Mythe et pensée chez les Grecs, Paris, $1971^{2}$ (1965), I, p. 13- 41 et «Le mythe hésiodique des races. Sur un essai de mise au point », ibid. p. 42-79. À ces études font pendant les analyses développées par Jean RUDHARDT, «Le mythe hésiodique des races et celui de Prométhée. Recherche des structures et des significations ", $D u$ mythe, de la religion grecque et de la compréhension d'autrui, Revue européenne des Sciences Sociales, t. 19, $\mathrm{n}^{\circ} 58$ (1981), p. 245-281.

44 RudHARDT, o.c. (n. 1), p. 118.

45 U.E. PAOLI, Studi sul processo attico, Milan, 1933, p. 67-71; L. GERNET, « Sur la notion de jugement en droit grec », in o.c. (n. 12), p. 61-81. 
parti pour l'un des deux plaideurs qui sont venus se présenter devant eux et exposer leur différend. Ce qui revient à proclamer la victoire de l'une des deux parties en présence, une fois qu'elles se sont affrontées dans le cadre d'une procédure judiciaire qui se déroule comme une sorte de duel, une eris, disait Paoli, un agôn, disait Gernet, au terme duquel il y a un gagnant et un perdant. La dikè émise par le ou par les juges désigne celui des deux adversaires qui se trouve dans son bon droit. Cette décision qui, dans les tribunaux athéniens de l'époque classique, s'obtient par un vote secret, est dans le monde d'Homère et d'Hésiode, dans ce monde de l'époque géométrique, publiquement formulée par les juges qui appartiennent à l'élite sociale, à la catégorie des « rois », des basileis.

Dans la scène judiciaire qui se trouve figurée sur le bouclier d'Achille ${ }^{46}$, deux hommes, le meurtrier et un parent de la victime, ont ainsi accepté de soumettre leur litige, qui porte sur le versement de la compensation, de la poinè destinée à compenser le meurtre, au jugement des Anciens. Ces Gerontes siègent publiquement au milieu de l'agora, entourés d'une foule venue assister à l'audience. Une fois que les deux adversaires ont parlé, les Anciens se lèvent et « chacun à leur

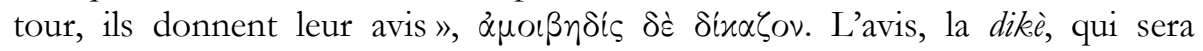
retenue sera celle qui aura été considérée par tout le monde, par l'ensemble des

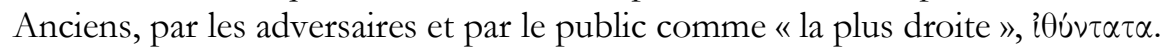

Même procédure lors de la querelle qui oppose Ménélas au jeune Antiloque qui a usé de ruse pour dépasser le roi de Sparte dans la course de chars et lui prendre la deuxième place. Lorsqu'il entend Antiloque revendiquer le second prix, Ménélas laisse éclater sa colère et en appelle au jugement des Anciens. Puis il se ravise, de crainte qu'on aille dire que ces Anciens lui ont été favorables en

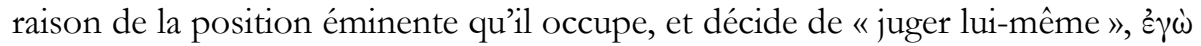
$\delta \varkappa \dot{\alpha} \sigma \omega$, en promettant que son arrêt (avec dikè qui est sous-entendue) sera « droit », i $i \varepsilon \tilde{i} \alpha^{47}$. Le souci formulé par le roi de Sparte de garantir la rectitude du jugement qu'il va prononcer renvoie à une réalité que dénonce Hésiode, dans des termes qui sont bien connus. Le poète d'Askra s'en prend en effet de manière répétée aux juges corrompus, les $\beta \alpha \sigma \iota \lambda \tilde{\eta} \varepsilon \varsigma \delta \omega \varrho o \varphi \alpha ́ \gamma o l$, les « rois mangeurs de présents $»^{48}$ qui prononcent des «sentences torses, $\sigma \chi 0 \lambda \iota \alpha i \operatorname{\delta ix} \alpha \iota »^{49}$. Il

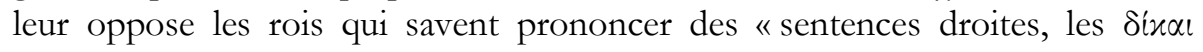

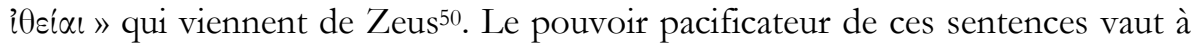
ceux qui savent en user d'être honorés comme des dieux ${ }^{51}$, tant est grande

\footnotetext{
46 Hom., Il. XVIII, 497-508.

47 Hom., Il. XXIII, 579-580.

48 Hés., Trav., 38-39; 221; 263-264.

${ }^{49}$ Hés., Trav., 221, 250, 264.

${ }^{50}$ Hés., Trav., 36; H.h.Dém., 152.

51 Hés., Trav., 86; 91-92 et 96.
} 
l'attente que suscitent parmi les hommes ces juges qui ont la charge d'user correctement de la dikè pour que le «juste », le dikaion, soit rétabli et respecté.

Il s'avère que le parcours effectué par Jean Rudhardt qui s'était engagé dans les différentes pistes que suggérait le classement proposé par les dictionnaires, sans en négliger aucune, a fait émerger une cohésion dont la notion de dikè semblait au premier abord dépourvue. Depuis la première série d'emplois dans laquelle dikè se trouve utilisée pour désigner «ce qui revient de droit» à un individu ou à un groupe d'individus, en passant par les usages qui caractérisent la dikè, « objective » ou « subjective », laquelle sert alors à rétablir ou à préserver un équilibre entre les éléments d'un même ensemble social, jusqu'aux sentences émises par les juges qui permettent, en cas de conflit, de désigner celui des deux adversaires qui est dans son droit. On pourrait conclure, sans risquer de trop dépasser la pensée du chercheur, qu'à travers ses mutiples attestations, dikè semble en définitive remplir un seul et même rôle, celui d'être un moyen au service d'une fin. Cette fin étant le maintien ou le rétablissement du dikaion, « une idée, une notion du juste » qui constitue ce que les Grecs entendent par justice.

La prise en compte des données les plus anciennes de la tradition éclaire par ailleurs la logique selon laquelle la pensée mythique fonctionne. Les données du mythe, telles qu'elles sont énoncées par Hésiode font en effet de Dikè la fille de Zeus. Ce faisant, elles établissent un lien étroit de parenté entre la jeune déesse et Zeus au point de faire de cette puissance une « collaboratrice » de son père, une force qui se dégage du dieu dont l'intelligence domine tout, une fonction qui dépend de lui et se trouve à son service. D'une certaine manière l'analyse du mythe donne des résultats semblables à ceux qui émanent de l'analyse lexicologique. Le mythe permet en l'occurrence de penser la complexité qui caractérise souvent l'enchaînement des événements, la succession des causes et des conséquences ainsi que les dysfonctionnements qui affectent les relations humaines. «L'exigence de justice, les manifestations de justice dans l'histoire sont liées à l'action du dieu qui assure l'équilibre du monde et qui en garantit l'ordonnance $»^{52}$. À côté de la conclusion qui est celle de l'historien des religions, un autre élément émerge cependant et éclaire ce qui relevait sans aucun doute de la conviction profonde de l'homme. L'idée qu'il y a malgré tout chez les humains une notion innée de la justice qui leur permet de reconnaitre la Dikè et qui confère à cet agent une efficacité privilégiée.

Évelyne SCHEID-TISSINIER

Université de Paris 13

Département d'Histoire

99, av. Jean-Baptiste Clément

F - 93430 Villetantanse

Courriel:evelyne.scheid@wanadoo.fr

${ }^{52}$ RUdhardT, o.c. (n. 1), p. 160. 